\title{
Shock Wave Response of Porous Materials: From Plasticity to Elasticity
}

\author{
Aiguo Xu, Guangcai Zhang, Yangjun Ying, Ping Zhang and \\ Jianshi Zhu \\ National Key Laboratory of Computational Physics, \\ Institute of Applied Physics and Computational Mathematics, P. O. Box 8009-26, \\ Beijing 100088, P.R.China \\ E-mail: Xu_Aiguo@iapcm.ac.cn
}

\begin{abstract}
Shock wave reaction results in various characteristic regimes in porous material. The geometrical and topological properties of these regimes are highly concerned in practical applications. Via the morphological analysis to characteristic regimes with high temperature, we investigate the thermodynamics of shocked porous materials whose mechanical properties cover a wide range from hyperplasticity to elasticity. It is found that, under fixed shock strength, the total fractional area $A$ of the high-temperature regimes with $T \geq T_{t h}$ and its saturation value first increase, then decrease with the increasing of the initial yield $\sigma_{Y 0}$, where $T_{t h}$ is a given threshold value of temperature $T$. In the shock-loading procedure, the fractional area $A(t)$ may show the same behavior if $T_{t h}$ and $\sigma_{Y 0}$ are chosen appropriately. Under the same $A(t)$ behavior, $T_{t h}$ first increases then decreases with $\sigma_{Y 0}$. At the maximum point $\sigma_{Y 0 M}$, the shock wave contributes the maximum plastic work. Around $\sigma_{Y 0 M}$, two materials with different mechanical properties may share the same $A(t)$ behavior even for the same $T_{t h}$. The characteristic regimes in the material with the larger $\sigma_{Y 0}$ are more dispersed.
\end{abstract}




\section{Introduction}

Porous materials are ubiquitous in nature and extensively used as industrial materials. Examples are referred to wood, bricks, metals, foams, ceramics, carbon and explosives. The use of porous materials in parts may lead to reduced weight, improved structural and mechanical properties, better heat transfer, greater motion and deformation control, etc 1, 2]. Besides others, they have also been used in surgical implant design to fabricate devices to replace or augment soft and hard tissues 3, 4. Although the study on shock wave reaction on porous materials has a long history. Most of previous studies were focussed on the global behaviors, such as the Hugoniots [5, 6, 7, 8, 9, 10, 11, 12] and the equation of state[13, 14, 15]. The dynamical procedures involve much richer physical behaviors but in fact are much less extensively investigated.

The main challenges for studying the dynamical behaviors in shocked porous material are twofold: the first is the numerical tool, the second is the scheme to analyze the simulation data. From the simulation side, an appropriate simulation tool must overcome two constraints. The first is the scale limitation. Molecular dynamics can discover some atomistic mechanisms of shock-induced void collapse[16, 17], but the spatial and temporal scales it may cover are far from being comparable with experiments. To overcome the scale limitation, one solution is to develop some mesoscopic particle methods. The second constraint is the numerical stability. Traditional simulation methods, both the Eulerian and Lagrangian ones, when treating with the dynamics of structured and/or porous materials, encountered severe difficulties. The reason is that material under investigation is generally highly distorted during the collapsing of cavities. The Eulerian description is not convenient for tracking interfaces. Lagrangian formulation has to rezone the meshes to restore proper shapes. The mapping of state fields of mass density, velocities and stresses from the distorted mesh to the newly generated one is not easy and introduces errors. In this study we use a mixed method, material-point method(MPM)[18, 19, to study shock wave reaction on porous materials. As a step to approach the shock wave dynamics in porous materials, we have carefully studied the cavity collapse in shocked materials [19, 20].

As for the second challenge, data analysis and information picking up, a relatively straightforward way is to study the local averaged values and the corresponding fluctuations of state variables[21]. In Ref.[21], the evolution of local turbulence mixing and volume dissipation were also studied. Shock wave reaction results in various characteristic regimes in porous materials, for example, regimes with high temperature, regimes with high pressure, regimes with high particle speeds, etc. These characteristic regimes are generally highly concerned in practical applications. Regimes with high temperature are places where initiation may start in energetic materials. Regimes with high pressure are places where phase transition may occur. Regimes with high particle speed are places where jet phenomena may occur. To understand the characteristic regimes defined by $\Theta \geq \Theta_{t h}$, we 22 introduced the Minkowski functionals to measure their morphological behaviors, where $\Theta$ is a physical variable under consideration, like 
the temperature, density, some specific stress, particle velocity or its components, $\Theta_{t h}$ a given threshold value.

Previous studies showed that the porous metal aluminum(Al) 22] and porous HMXlike material[23] show significant differences under shock wave reaction. To clarify the effects of single material parameters and present indicative information for material designs, it is interesting to have a through study on the shock behaviors in relation to their mechanical property ranging from hyper-plastic to pure elastic 24.

In present paper we focus on characteristic regimes with high temperature. We organize the following part of the paper as follows. Section 2 briefly introduces the material model and the numerical method. Section 3 outlines the morphological characterization for characteristic regimes manifested by Turing patterns. Simulation results are shown and analyzed in section 4 . Section 5 makes the conclusion.

\section{Material model and material-point method}

The porous material is fabricated by a solid body with a number of randomly distributed voids embedded. The solid body follows an associative von Mises plasticity model with linear kinematic and isotropic hardening[21]. The pressure $P$ is calculated by using the Mie-Grüneissen equation of state which can be written as $P-P_{H}=$ $[\gamma(V) / V]\left[E-E_{H}\left(V_{H}\right)\right]$, where $P_{H}, V_{H}$ and $E_{H}$ are pressure, specific volume and energy on the Rankine-Hugoniot curve, respectively. The relation between $P_{H}$ and $V_{H}$ can be estimated by experiments and be written as

$$
P_{H}= \begin{cases}\frac{\rho_{0} c_{0}^{2}\left(1-\frac{V_{H}}{V_{0}}\right)}{(\lambda-1)^{2}\left(\frac{\lambda}{\lambda-1} \times \frac{V_{H}}{V_{0}}-1\right)^{2}}, & V_{H} \leq V_{0} \\ \rho_{0} c_{0}^{2}\left(\frac{V_{H}}{V_{0}}-1\right), & V_{H}>V_{0}\end{cases}
$$

where $\rho_{0}, V_{0}$ are the initial density and specific volume of the solid material, $c_{0}$ the sound speed, $\lambda$ the coefficient in Hugoniot velocity relation. Both the shock compression and the plastic work $E-E_{H}\left(V_{H}\right)$ cause the increasing of temperature. The increasing of temperature from shock compression can be calculated as:

$$
\frac{\mathrm{d} T_{H}}{\mathrm{~d} V_{H}}=\frac{c_{0}^{2} \cdot \lambda\left(V_{0}-V_{H}\right)^{2}}{c_{v}\left[(\lambda-1) V_{0}-\lambda V_{H}\right]^{3}}-\frac{\gamma(V)}{V_{H}} T_{H} .
$$

where $c_{v}$ is the specific heat. Eq.(2) can be derived from the thermal equation and the Mie-Grüneissen equation of state[21]. The increasing of temperature from plastic work can be calculated as: $\mathrm{d} T_{p}=\mathrm{d} W_{p} / c_{v}$.

We model shocked materials with continuously varying mechanical properties. The reference material is the metal aluminum. The corresponding parameters are as below: density in solid portion $\rho_{0}=2700 \mathrm{~kg} / \mathrm{m}^{3}$, Yang's module $E=69 \mathrm{Mpa}$, Poisson's ratio $\nu=0.33$, initial yield $\sigma_{Y 0}=120 \mathrm{Mpa}$, tangential module $E_{\tan }=384 \mathrm{MPa}$, sound speed $c_{0}=5.35 \mathrm{~km} / \mathrm{s}$, characteristic coefficient in the Hugoniot velocity relation $\lambda=1.34$, specific heat $c_{v}=880 \mathrm{~J} /(\mathrm{Kg} \cdot \mathrm{K})$, heat conduction coefficient $k=237 \mathrm{~W} /(\mathrm{m} \cdot \mathrm{K})$, and Grüneissen coefficient $\gamma=1.96$. The initial temperature of the material is $300 \mathrm{~K}$. In simulations a wide range of the yield $\sigma_{Y 0}$ will be used. 
The material point method is a relatively new particle method in computational solid mechanics. This method uses a regular structured grid as a computational scratchpad for computing spatial gradients of field variables. The grid is convected with the particles during deformations that occur over a time step, eliminating the diffusion problems associated with advection on an Eulerian grid. The grid is restored to its original location at the end of a time step. In addition to avoiding the Eulerian diffusion problem, this approach also circumvents problems with mesh entanglement that can plague fully Lagrangian-based techniques when large deformations are encountered. The MPM has also been successful in solving problems involving impact, etc. It has an advantage over traditional finite element methods in that the use of the regular grid eliminates the need for doing costly searches for contact surfaces. Details of the scheme is referred to our previous publications [19, 18].

\section{Outline of morphological description}

A variety of techniques can be used to describe the complex spatial distribution and time evolution of physical quantities in the shocked porous material. In this study we concentrate on the set of statistics known as Minkowski functionals [25, 26, 27]. The Minkowski functionals has been successfully used to characterize patterns in reaction-diffusion systems [28], spinodal decomposition [29, 30], fluctuations of cosmic microwave background 31], block copolymer systems 32, 33] and to reconstruct complex materials 34$]$.

Assume $\Theta$ is a physical quantity being interesting to us, then the regions with $\Theta \geq \Theta_{t h}$ in the shocked porous material are referred to as characteristic regimes, where $\Theta_{t h}$ is a threshold value of $\Theta$. To simplify the analysis of the complex physical field, we first condense the physical field as two kinds of characteristic regimes, the white and the black. The white correspond to regimes with $\Theta \geq \Theta_{t h}$ and the black correspond to regimes with $\Theta<\Theta_{t h}$. For such Turing patterns, a general theorem of integral geometry states that all properties of a $d$-dimensional convex set which satisfy motion invariance and additivity (called morphological properties) are contained in $d+1$ numerical values [35]. For a condensed temperature field, the white correspond to the high-temperature regimes and the black correspond to the low-temperature regimes. The high-temperature regimes are also generally referred to "hot-spots".

For a two-dimensional temperature map, the three Minkowski functionals correspond geometrically to the total fractional area $A$ of the high-temperature regimes, the boundary length $L$ between the high- and low-temperature regimes regimes per unit area, and the Euler characteristic $\chi$ per unit area (equivalent to the topological genus). When we increase the temperature threshold $T_{t h}$ from the lowest temperature to the highest one, the high-temperature area $A$ will decrease from 1 to 0 ; the boundary length $L$ first increases from 0 , then arrives at a maximum value, finally decreases to 0 again. There are several ways to define the Euler characteristic $\chi$. Two simplest one is $\chi=\left(N_{W}-N_{B}\right) / N$, where $N_{W}\left(N_{B}\right)$ is the number of connected white (black) 
Figure 1. (Color online) Snapshots of shocked porous material, where $\Delta=0.5$, $v_{\text {init }}=1000 \mathrm{~m} / \mathrm{s}, \mathrm{t}=1000 \mathrm{~ns}$. From left to right and from top to bottom, the initial yields in the two rows are $\sigma_{Y 0}=12,120,3000,8000,10000,12000,15000,20000 \mathrm{Mpa}$, respectively. The unit of length is $10 \mu \mathrm{m}$. The width and height of the simulated system are $1 \mathrm{~mm}$ and $5 \mathrm{~mm}$, respectively. From blue to red the color corresponds to increase of temperature.

regimes, $N$ is the total number of pixels. In contrast to the area $A$ and boundary length $L$, the Euler characteristic $\chi$ describes the connectivity of the characteristic regimes in the material. It describes the patterns in a purely topological way, i.e., without referring to any kind of metric. It is negative (positive) if many disconnected black (white) regimes dominate the image. A vanishing Euler characteristic indicates a highly connected structure with equal amount of black and white regimes. The ratio $\kappa=\chi / L$ describes the mean curvature of the boundary line separating black and white regimes. More discussions and calculation schemes of the Minkowski functionals are referred to Refs. [26, 28, 36].

Among the three Minkowski functionals, the high-temperature area $A$ is the only one which monotonically increases in the shock-loading procedure and/or when the threshold value becomes smaller. For a given $T_{t h}$, its increasing rate, $D$, presents meaningful information. When the temperature threshold $T_{t h}$ becomes higher, $D$ decreases. The variations of $A, L$ and $\chi$ with $T_{t h}$ and time $t$ compose a scenario for the shock response of porous material[22].

\section{Simulation results and physical interpretation}

If denote the mean density of the porous body as $\rho$, then the porosity of the material is $\Delta=1-\rho / \rho_{0}[37$. The shock to target body is loaded by colliding with a rigid wall being static at the bottom position $y=0$. The initial velocity of the porous body is $-v_{\text {init }}$. The collision starts at time $t=0$. The height and width of the porous body are $5 \mathrm{~mm}$ and $1 \mathrm{~mm}$, respectively. Periodic boundary conditions are used in the horizontal directions, which means that the real system is composed of many of the simulated ones aligned periodically in the horizontal direction.

\subsection{Case with $v_{\text {init }}=1000 \mathrm{~m} / \mathrm{s}$}

Figure 1 presents a series of snapshots in the shock loading procedure, where the porosity $\Delta=0.5$ and initial velocity $v_{\text {init }}=1000 \mathrm{~m} / \mathrm{s}$, the time $t=1000 \mathrm{~ns}$. From left to right and from top to bottom, the two rows of snapshots are for cases with $\sigma_{Y 0}=12,120$, 3000, 8000, 10000, 12000, 15000, $20000 \mathrm{Mpa}$, respectively. The points in the figure correspond to the material particles. The color from blue to red corresponds to increasing of temperature. From Fig.1 one can find the moving down of upper free surface and moving up of global compressive-waves series. For each case, the initial shock wave is decomposed as a complex series of compressive and rarefactive waves. In the shock 


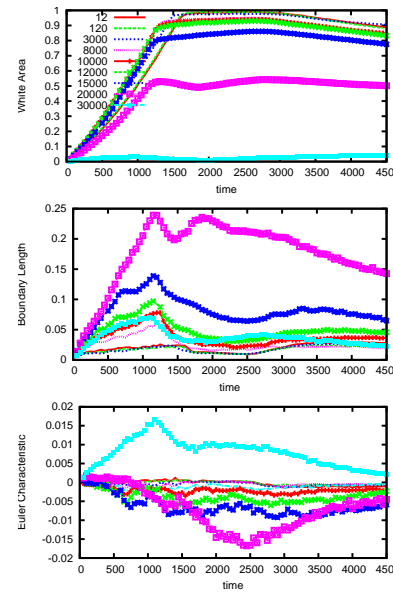

(a)
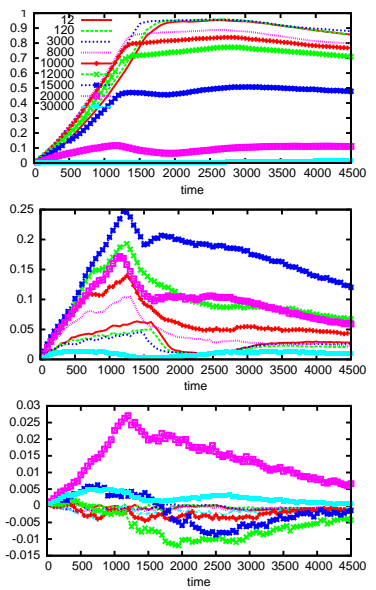

(b)
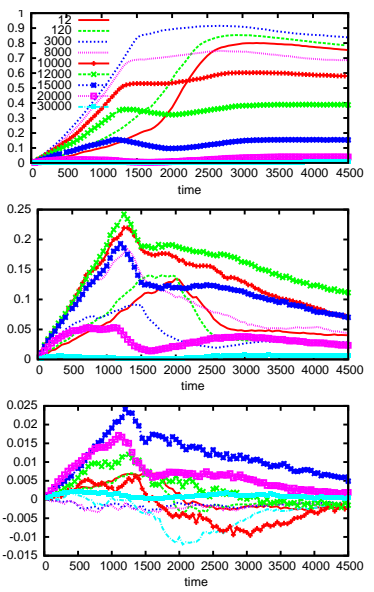

(c)

Figure 2. (Color online) Minkowski measurements for cases with various initial yields, where $\Delta=0.5, v_{\text {init }}=1000 \mathrm{~m} / \mathrm{s}$. The values of $\sigma_{Y 0}$ are shown in the legend with the unit MPa. $T_{t h}=400 \mathrm{~K}$ in (a), $T_{t h}=500 \mathrm{~K}$ in (b), $T_{t h}=600 \mathrm{~K}$ in (c).

loading procedure, the compressive effects dominate. Within the shocked region, both the plastic work and shock compression make the temperature increase. In the present case, the plastic work dominates. The high-temperature regimes in materials with higher initial yields are more dispersed. The increasing rate of high-temperature area $A(t)$ is different when the initial yield changes.

To quantify and get a more complete understanding on the shock wave response behavior, we show a set of morphological measures versus time in Fig.2(a), where values of initial yields are shown in the legend. The temperature threshold here is $T_{t h}=400$ $\mathrm{K}$. With the propagation of compressive-wave-series in the porous material, the hightemperature area $A$ first increases with time in a parabolic way, then approaches to a saturation value slowly, keeps the saturation value for a period, finally decreases. The final decrease indicates that rarefactive waves are reflected back from the upper free surface and are decreasing the mean pressure and temperature, an amount of material particles changed their temperature from $T>T_{t h}$ to $T<T_{t h}$. During the saturation period, more compressive waves arrived at the upper free surface, consequently, more rarefactive waves are reflected back into the porous body. The former tends to increase the temperature, while the latter tends to decrease. The two effects are nearly balanced. Therefore, the high-temperature area $A$ keeps nearly a constant.

As for effects of the initial yield $\sigma_{Y 0}$, we can find two interesting phenomena: Both the initial increasing rate and the saturation value of $A$ first increase, then decrease when the material changes from being superplastic to pure elastic. For cases checked in our numerical experiments, the increasing rate $D$ becomes larger when the initial yield $\sigma_{Y 0}$ increases from 0 to about $10 \mathrm{Gpa}$. If $\sigma_{Y 0}$ further to increase, $D$ will decrease. The saturation value $A_{S}$ of high-temperature area becomes larger when $\sigma_{Y 0}$ increases from being very small to about $1 \mathrm{Gpa}$. When $\sigma_{Y 0}$ becomes larger, the saturation value $A_{S}$ 
decreases. For the time interval shown Fig. 2(a), when $\sigma_{Y 0}>10 \mathrm{Gpa}$, the saturation value $A_{S}$ decreases and the period for $A \approx A_{S}$ becomes shorter with the increasing of $\sigma_{Y 0}$. When $\sigma_{Y 0}=12 \mathrm{Gpa}, A \approx 0.96$ during the period $1430 \mathrm{~ns}<t<2924 \mathrm{~ns}$; When $\sigma_{Y 0}=15 \mathrm{Gpa}, A$ increases slowly from 0.8 to 0.86 during the period $1245 \mathrm{~ns}$ $<t<2825 \mathrm{~ns}$; When $\sigma_{Y 0}=20 \mathrm{Gpa}, A_{S} \approx 0.52$ and have a local minimum value 0.49 at about $t=1863 \mathrm{~ns}$. When $\sigma_{Y 0}=30 \mathrm{Gpa}$, the area $A$ keeps very small.

Now we check the information given by the boundary length and Euler characteristic in Fig.2(a). For all cases shown in the figure, the boundary length $L$ first increases, then decreases with time. The former increase corresponds to the propagation of compressive waves and the appearance of more hot-spots. The decreasing of $L(t)$ is not monotonic. The initial decrease corresponds to the coalescence of some hot-spots, the latter decrease corresponds to the coming in of the global rarefactive waves from the upper free surface, which result in expanding and coalescence of some cold-spots. A prominent behavior here is that, for the case with $\sigma_{Y 0}=20 \mathrm{Gpa}$, the boundary length $L$ has the largest value. For this case, the value of $\chi$ changes from being slightly positive to being the most negative. Combining information of $A, L$ and $\chi$, we can know that, with the propagation of compressive wave in the porous material, the number of hot-spots with $T>400 \mathrm{~K}$ quickly increases, but distributes quite scatterredly. After corresponding compressive wave scanned all the material body, some scattered cold-spots with $T<400 \mathrm{~K}$ expand and partly coalescence due to the coming in of rarefactive waves. During this procedure, some small hot-spots with $T>400 \mathrm{~K}$ disappear. Therefore, both the high-temperature area $A$ and boundary length $L$ decrease and the Euler Characteristic $\chi$ becomes more negative. For other cases, the smaller the boundary length $L$, the flatter the wave front in the temperature map. The material with $\sigma_{Y 0}=15 \mathrm{Gpa}$ has the secondary maximum boundary length $L$ and more flatter $\chi(t)$ curve than the material with $\sigma_{Y 0}=20$ Gpa. This means that the numbers of hot-spots and cold-spots do not have much difference. The number of cold-spots dominates slightly during the time interval shown in the figure.

In the shock loading procedure, if we decrease the threshold value $T_{t h}$, the wave fronts in the pixelized temperature map becomes flater. Consequently, the values of $L(t)$ are smaller, the $\chi(t)$ values are closer to zero, and the curves for $A(t)$ becomes closer to be linear. If we increase the threshold value $T_{t h}$, the pixelized temperature map shows different geometric and topological behaviors. Examples are referred to Figs. 2 (b) and $2(\mathrm{c}) . T_{t h}=500 \mathrm{~K}$ in Fig. 2(b) and $T_{t h}=600 \mathrm{~K}$ in Fig. 2(c). It is clear that the saturation values of $A(t)$ decrease with the increasing of $T_{t h}$. In the shock-loading procedure, the material with $\sigma_{Y 0}=12 \mathrm{Mpa}$ has about $20 \%$ of material particles can not get a temperature higher than $600 \mathrm{~K}$ and $5 \%$ can not get the temperature higher than $500 \mathrm{~K}$, and only $1 \%$ can not get the temperature higher than $400 \mathrm{~K}$. For the material with $\sigma_{Y 0}=120 \mathrm{Mpa}$, in the shock loading procedure, there are about $15 \%$ of material particles can not get the temperature higher than $600 \mathrm{~K}, 4 \%$ can not get the temperature higher than $500 \mathrm{~K}$, and only $1 \%$ can not get the temperature higher than $400 \mathrm{~K}$.

When the initial yield is very high, for example, $\sigma_{Y 0}=15 \mathrm{Gpa}$, the material is very elastic. Consequently, the saturation value $A_{S}$ of high-temperature area is small. For 


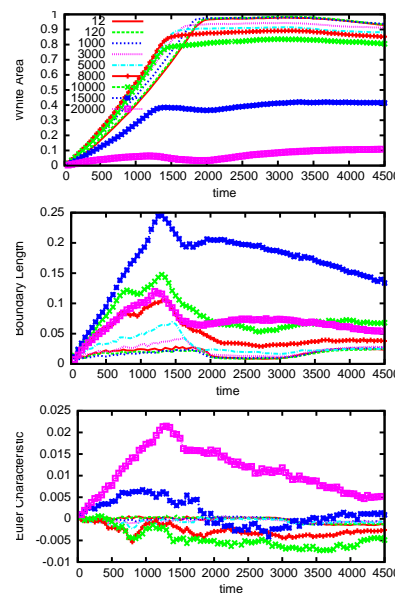

(a)
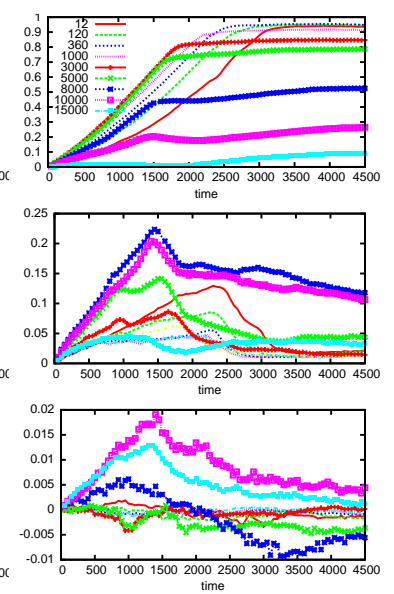

(b)
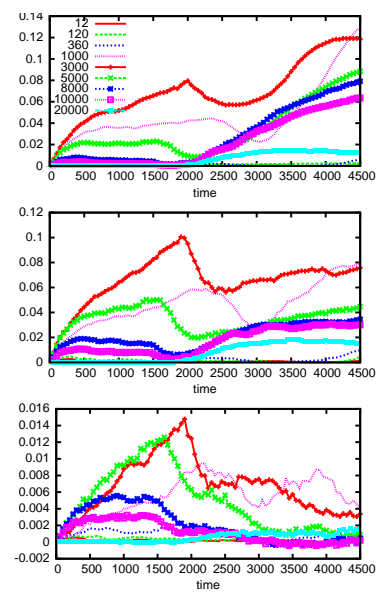

(c)

Figure 3. (Color online) Minkowski measurements for cases with various initial yields, where $\Delta=0.5$. The values of $\sigma_{Y 0}$ are shown in the legend with the unit MPa. The threshold temperature is $400 \mathrm{~K}$. $v_{\text {init }}=800 \mathrm{~m} / \mathrm{s}$ in $(\mathrm{a}), v_{\text {init }}=600 \mathrm{~m} / \mathrm{s}$ in (b), $v_{\text {init }}=400 \mathrm{~m} / \mathrm{s}$ in (c).

example, $A_{S}=0.15$ when $T_{t h}=600 \mathrm{~K}$, which means that $85 \%$ of material particles can not get the temperature higher than $600 \mathrm{~K}$. For the case with $\sigma_{Y 0}=20 \mathrm{Gpa}, A_{S}=0.54$ when $T_{t h}=400 \mathrm{~K}, A_{S}=0.11$ when $T_{t h}=500 \mathrm{~K}$ and $A_{S}=0.04$ when $T_{t h}=600 \mathrm{~K}$. For the case with $\sigma_{Y 0}=30 \mathrm{Gpa}$, only $0.1 \%$ of material particles can get a temperature higher than $600 \mathrm{~K}$ in the shock loading procedure. In the temperature pattern with $T_{t h}=400 \mathrm{~K}$, the case of $\sigma_{Y 0}=20 \mathrm{GPa}$ has a largest boundary length. When $T_{t h}=500 \mathrm{~K}$, the case of $\sigma_{Y 0}=15 \mathrm{GPa}$ has a largest boundary length. When $T_{t h}=600 \mathrm{~K}$, the case of $\sigma_{Y 0}=10 \mathrm{GPa}$ has the largest $L$.

\subsection{Effects of initial shock strength}

With the decreasing of initial shock strength, both the highest and the mean temperatures in the shocked portion decrease. A set of Minkowski measures for the shocking procedure of porous materials with various initial yields are shown in Fig.3, where $\Delta=0.5$ and $T_{t h}=400 \mathrm{~K}$. The initial impact velocities are different in Figs. (a), (b) and (c). They are $800 \mathrm{~m} / \mathrm{s}, 600 \mathrm{~m} / \mathrm{s}$ and $400 \mathrm{~m} / \mathrm{s}$, respectively. The values of initial yields are shown in the legends. Specifically, the initial yields are 12, 120, 1000,3000, 5000, 8000, 10000, 15000 and 20000MPa in Fig.3(a) ; in Fig.3(b) they are 12, 120, 360, 1000,3000, 5000, 8000, 10000 and 15000MPa; in Fig.3(c) they are 12, 120, 360, 1000,3000, 5000, 8000, 10000, and 20000MPa. Compared with cases shown in Fig. 2(a), both the saturation value $A_{S}$ and the increasing rate $D\left(T_{t h}\right)$ of the high-temperature area decrease when the initial shock becomes weaker. As an example, for the material with $\sigma_{Y 0}=1000 \mathrm{Mpa}$, when the initial impact velocity is $v_{\text {init }}=1000 \mathrm{~m} / \mathrm{s}$, there are $99 \%$ of material particles arrive at the temperature higher than $400 \mathrm{~K}$ in the shockloading procedure; when $v_{\text {init }}=800 \mathrm{~m} / \mathrm{s}$, the fraction of material particles getting the 

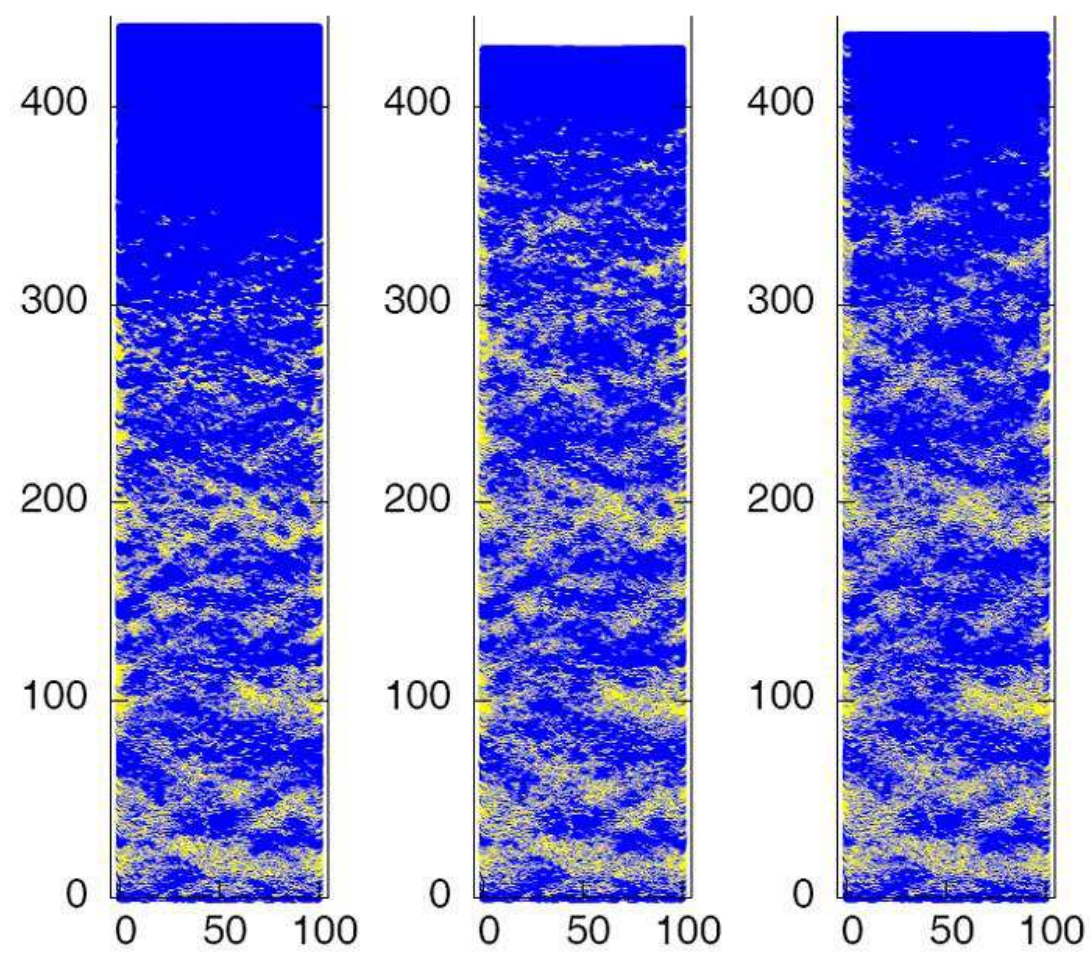

Figure 4. (Color online) Configurations with condensed temperature map, where $\Delta=0.5, v_{\text {init }}=400 \mathrm{~m} / \mathrm{s}, \sigma_{Y 0}=3000 \mathrm{Mpa}$. The areas with temperature higher than $400 \mathrm{~K}$ are shown in yellow, other areas are in blue. The times corresponding to the three snapshots are 1500ns, 2000ns and 2500ns, respectively, from which one can find that the high-temperature area at $\mathrm{t}=2000 \mathrm{~ns}$ is a little larger than that at $\mathrm{t}=2500 \mathrm{~ns}$ and that at $\mathrm{t}=1500 \mathrm{~ns}$.

temperature higher than $400 \mathrm{~K}$ is $97 \%$; when $v_{\text {init }}=600 \mathrm{~m} / \mathrm{s}$, the fraction is $89 \%$; when $v_{\text {init }}=400 \mathrm{~m} / \mathrm{s}$, the fraction becomes only $5 \%$.

In Fig.3(c), the case of $\sigma_{Y 0}=3000 \mathrm{MPa}$ has the maximum high-temperature area $A$, boundary length $L$ and Euler characteristic $\chi$. In this figure, only after the hightemperature area $A$ gets its maximum value, it begins to decrease, which means that, under such a shock strength, most material particles can not get a temperature higher than $400 \mathrm{~K}$, the threshold value $400 \mathrm{~K}$ has been very close to the highest temperature in this system. To understand better why the maximum high-temperature area occurs at about $\mathrm{t}=2000 \mathrm{~ns}$, we show the configurations with condensed temperature map in Fig.4, where the three snapshots are for the times $\mathrm{t}=1500 \mathrm{~ns}, 2000 \mathrm{~ns}$ and $2500 \mathrm{~ns}$, respectively. The areas with temperature higher than $400 \mathrm{~K}$ are shown in yellow, other areas are shown in blue. One can find that the high-temperature area at time $t=2000 \mathrm{~ns}$ is the largest among the three snapshots. From the heights of the upper free surface, one can find that the one for $t=2000 \mathrm{~ns}$ is the lowest, which means that the shock-loading procedure finishes at about that time. When the unloading procedure starts, the area with hightemperature decreases. From Fig.4 one can also find that the high-temperature regimes for $T_{t h}=400 \mathrm{~K}$ have been very dispersed. This is consistent with the large value of boundary length, and consistent with the above observation that only a small portion of 

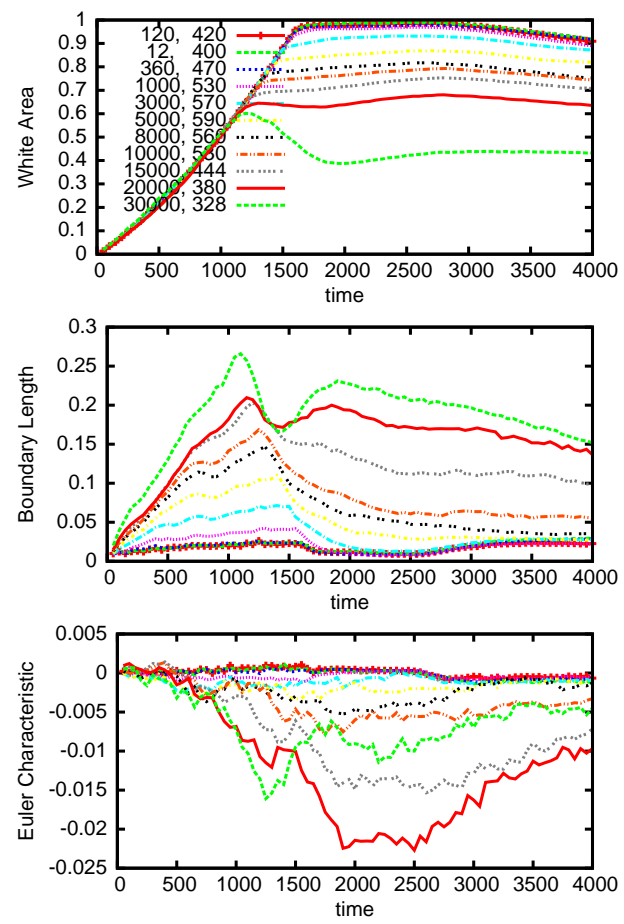

Figure 5. (Color online) Minkowski measurements versus time, where $\Delta=0.5$, $v_{\text {init }}=1000 \mathrm{~m} / \mathrm{s}$, the values of $\sigma_{Y 0}$ and $T_{t h}$ are shown in the two columns of the legend. The units are $\mathrm{MPa}$ and $\mathrm{K}$, respectively.
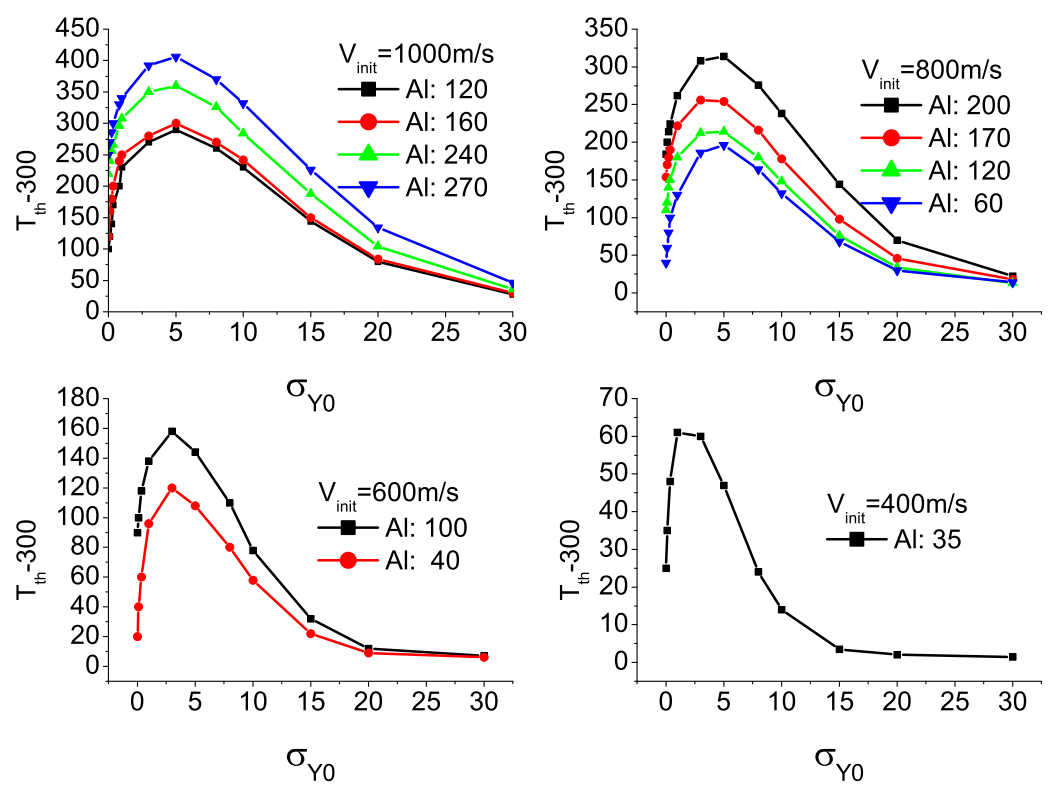

Figure 6. (Color online) $T_{t h}$ versus $\sigma_{Y 0}$ for the same $A(t)$ behavior. The initial impact velocities are shown in the legends. Each curve in the figure is labeled by the $T_{t h}$ value for the reference material, aluminum $(\mathrm{Al})$. The units for the horizontal and vertical axes are GPa and $\mathrm{K}$, respectively. 
material particles get the temperature higher than 400K under such a shock strength.

\section{3. $\left(\sigma_{Y 0}, T_{t h}\right)$ pairs for the same $A(t)$ behavior}

As mentioned above, among the three Minkowski functionals, the high-temperature area $A(t)$ is the only one being monotonic when the threshold value $T_{t h}$ decreases and/or with the going on of the shock-loading procedure. It is natural to check if $A(t)$ shows the same behavior when using appropriate $\left(\sigma_{Y 0}, T_{t h}\right)$ pairs. Figure 5 shows such examples for the case with initial impact velocity $v_{\text {init }}=1000 \mathrm{~m} / \mathrm{s}$. In Fig.5, the temperature threshold for reference material, aluminum, is 420K. From Fig. 5 we can find that materials with different initial yields, if we choose an appropriate $T_{t h}$ to observe, the high-temperature area $A(t)$ shows the same behavior in the shock-loading procedure. Such a property can be understood better by observing Figs. 1 and 4 . From Figs. 1 and 4, it is also clear that, for a fixed shock strength, with the increasing of the initial yield, the wave front becomes wider, the high-temperature regimes becomes more scattered, more low-temperature domains are embedded in the compressed portion. This morphological behavior is manifested by larger boundary lengths and more negative Euler characteristics in Fig.5.

If use the $\left(\sigma_{Y 0}, T_{t h}\right)$ pairs in Fig.5 as coordinates, we get the curve labeled by " $V_{\text {init }}=1000 \mathrm{~m} / \mathrm{s}$ " and "Al: 120" in Fig.6. For the shock strength $v_{\text {init }}=1000 \mathrm{~m} / \mathrm{s}$, the material with $\sigma_{Y 0}=5 \mathrm{GPa}$ has the maximum $T_{t h}$ which is about $590 \mathrm{~K}$. In this case, the shock contributes the maximum plastic work and the system has the highest temperature. If increase the temperature threshold $T_{t h}$ to $460 \mathrm{~K}, 540 \mathrm{~K}$ and $570 \mathrm{~K}$, we have the other three curves. Along each of them, $A(t)$ shows the same behavior in the shock-loading procedure. If decrease the shock strength to $v_{\text {init }}=800 \mathrm{~m} / \mathrm{s}, 600 \mathrm{~m} / \mathrm{s}$ and $400 \mathrm{~m} / \mathrm{s}$, we get the other three plots of Fig.6. The maximum value point $\sigma_{Y 0 M}$ moves towards the lower value of the initial yield when the shock wave becomes weaker. In the shock loading procedure, if we decrease the threshold value $T_{t h}$, the wave fronts becomes flater. Consequently, $L(t)$ curves become smaller, $\chi(t)$ becomes closer to zero; the curves for $A(t)$ becomes closer to be linear.

\section{Conclusion}

Shock wave reaction results in various characteristic regimes in porous materials. The properties of these regimes are highly concerned in practical applications. Based on the material-point simulation and morphological characterization, we investigate how the initial yield influence the behavior of high temperature regimes in shocked porous material. It is found that, under fixed shock strength, the total fractional area $A$ of hightemperature regimes (with $T \geq T_{t h}$ ) and its saturation value first increase, then decrease when initial yield $\sigma_{Y 0}$ becomes higher. In the shock-loading procedure the fractional area $A(t)$ may show the same behavior under various choices of $T_{t h}$ and $\sigma_{Y 0}$. For the same $A(t)$ behavior, $T_{t h}$ first increases then decreases when $\sigma_{Y 0}$ becomes higher. At the maximum 
point $\sigma_{Y 0 M}$, the plastic work by the shock gets the maximum value. Around $\sigma_{Y 0 M}$, two materials with different mechanical properties may share the same $A(t)$ behavior even for the same threshold $T_{t h}$. The high-temperature regimes in the material with the higher initial yield $\sigma_{Y 0}$ are more dispersed. Other kinds of characteristic regimes, for example, those with high pressure, high particle speed, etc., can be studied in the same way.

\section{Acknowledgments}

A. $\mathrm{Xu}$ is grateful to Prof. Hua Li for helpful discussions on shock waves and porous materials, to Drs. G. Gonnella, A. Lamura and V. Sofonea for helpful discussions on morphological description. This work is supported partly by Science Foundations of Laboratory of Computational Physics, China Academy of Engineering Physics [under Grant Nos. 2009A0102005, 2009B0101012], and National Science Foundation of China (under Grant Nos.10702010, 10775018 and 10604010).

\section{References}

[1] S. Nemat-Nasser and M. Hori, Micromechanics: Overall Properties of Heterogeneous Materials Second Revised Edition, Elsevier Science Press, 1999.

[2] V. F. Nesterenko, Dynamics of Heterogeneous Materials, Springer-Verlag, New York, 2001.

[3] M. Lundberg, B. Skårman, F. Cesar, L. R. Wallenberg, Microporous and Mesoporous Materials, 5497 (2002).

[4] G. Lu, G.Q.M. Lu, and Z.M. Xiao, J. Porous Materials 6, 359 (1999).

[5] R. K. Linde and D. N. Schmidt, J. Appl. Phys. 37, 3259 (1966).

[6] R. R. Boade, J. Appl. Phys. 40, 3781 (1969).

[7] B. M. Butcher, J. Appl. Phys. 45, 3864 (1974).

[8] S. Bonnan and P. L. Hereil, J. Appl. Phys. 83, 5741 (1998).

[9] G. T. Gray III, N. K. Bourne and J. C. F. Milett, J. Appl. Phys. 94, 6430 (2003).

[10] A. D. Resnyansky, N. K. Bourne, J. Appl. Phys. 95, 1760 (2004).

[11] D. J. Pastine, M. Lombardi, A. Chatterjee and W. Tchen, J. Appl. Phys. 41, 3144 (1970).

[12] L. Boshoff-Mostert and H. J. Viljoen, J. Appl. Phys. 86, 1245 (1999).

[13] Q. Wu and F. Jing, Appl. Phys. Lett. 67, 49 (1995).

[14] Q. Wu and F. Jing, J. Appl. Phys. 80, 4343 (1996).

[15] H. Geng, Q. Wu, H. Tan, L. Cai and F. Jing, J. Appl. Phys. 92, 5924 (2002).

[16] P. Erhart, E. M. Bringa, M. Kumar, and K. Albe, Phys. Rev. B 72, 052104 (2005).

[17] Q. Yang, G. Zhang, A. Xu, Y. Zhao, Y. Li, Acta Phys. Sini. 57, 940 (2008).

[18] X. F. Pan, A. Xu, G. Zhang, et al, Commun. Theor. Phys. 49, 1129 (2008).

[19] X. F. Pan, A. Xu ,G. Zhang and J. Zhu, J. Phys. D: Appl. Phys. 41, 015401 (2008).

[20] A. Xu, X. F. Pan, G. Zhang and J. Zhu, J. Phys.: Condens. Matter 19, 326212(2007).

[21] A. Xu, G. Zhang, X. F. Pan, J. Zhu, Commun. Theor. Phys. 51, 691 (2009).

[22] A. Xu, G. Zhang, X. F. Pan, P. Zhang and J. Zhu, J. Phys. D: Appl. Phys. 42, 075409 (2009).

[23] A. Xu, G. Zhang, P. Zhang, X. F. Pan, J. Zhu, Commun. Theor. Phys. 52, 901 (2009).

[24] Y. Jiang and M. Liu, Phys. Rev. Lett. 99, 105501 (2007); A. Xu, G. Zhang, H. Li and J. Zhu Chin. Phys. Lett. 27, 026201 (2010).

[25] H. Minkowski, Mathematische Annalen, 57 447(1903).

[26] K. R. Mecke, Int. J. Mod. Phys. B 12, 861 (1998).

[27] Hubert Mantz, Karin Jacobs and Klaus Mecke, J. Stat. Mech. P12015 (2008). 
[28] K. R. Mecke, Phys. Rev. E 53, 4794 (1996).

[29] K. R. Mecke and V. Sofonea, Phys. Rev. E 56, R3761 (1997).

[30] A. Aksimentiev, Krzysztof Moorthi and R. Holyst, J. Chem. Phys. 112, 6049 (2000).

[31] P. Cabella, F. Hansen, D. Marinucci, D. Pagano and N. Vittorio, Phys. Rev. D 69, 063007 (2004).

[32] G. J. A. Sevink and A. V. Zvelindovsky, J. Chem. Phys. 121, 3864 (2004)

[33] S. Rehse, K. Mecke, R. Magerle, Phys. Rev. E 77, 051805 (2008).

[34] C. H. Arns, M. A. Knackstedt and K. R. Mecke, Phys. Rev. Lett. 91, 215506 (2003).

[35] H. Hadwiger, Abh. Math. Sem. Univ. Hamburg 20, 136 (1956); Math. Z. 71, 124 (1959).

[36] V. Sofonea, et al, Eur. Phys. J. B 8, 99 (1999).

[37] In our previous paper 22, the porosity is described by the ratio $\delta=\rho_{0} / \rho$. The relation between $\Delta$ and $\delta$ is $\Delta=1-1 / \delta$. The larger $\delta$ is, the larger $\Delta$ is. 
This figure "Fig1.jpg" is available in "jpg" format from: http://arxiv.org/ps/1005.0908v1 